\title{
Analysis and Research of the Influence of Taxi Subsidy Scheme on Urban Taxi-Hailing Difficulties
}

\author{
Qiang Mengye ${ }^{1, a}$, Bian Zhengyang ${ }^{2}$, Liu Yongyan ${ }^{3}$ \\ ${ }^{1}$ Nanjing University of Posts and Telecommunications, Electrical and Computer Engineering, \\ jiangsu, nanjing, 210023 \\ ${ }^{2}$ Nanjing University of Posts and Telecommunications, Electrical and Computer Engineering, \\ jiangsu, nanjing, 210023 \\ ${ }^{3}$ Nanjing University of Posts and Telecommunications, Electrical and Computer Engineering, \\ jiangsu, nanjing, 210023 \\ aemail: dzy_nj@sina.com
}

Keywords: Taxi Software; Time Pricing; Evolutionary Game Theory Model; Co-Ordination Of Supply And Demand; Social Welfare Mode

\begin{abstract}
Difficulty in taking a taxi is a long term problem entangles with both customers and drivers, and taxi software was born under such background. These apps consider about both customers and drivers to analyze the contradiction between taxi taking and order receiving, then link the waiting time and subsidy profit together to assess the feasibility of current scheme, which will price by time and evolutionary game theory model to market. Finally, this article will promote a proper subsidy scheme based on social welfare theory.
\end{abstract}

\section{Introduction}

With economic development in China and improved modern construction, taxi comes to become one of daily transportation for citizens. The taxi market is different from ideal market in traditional economic analysis, and the supply and demand relationship almost depends on many intermediate variable such as taxi empty loading rate and time and space distribution differences,etc. This article will analyze taxi-call problem from two parts. Firstly, we will analyze the behaviors of customer and empty cabs influenced by current subsidy and choose taxi empty loading rate, taxi density and waiting time for driving as assessment index for taxi matching degree of supply and demand, which also will analyze the degree for peak time in morning, noon and evening of business area and higher education region.Then connect subsidy profit with taxi waiting time to reach the interval lose balance between short-term and long-term order orders, in the mean time, using evolutionary stable strategy and combine with social welfare theory to promote the best distribution ratio of subsidy.

\section{Subsidy Influences for Taxi Supply and Demand Relationship-Choose Taxi Drivers as the Object of Study}

\section{Behavior characteristics for taxi driver route choice}

Under the charge system of pay per $\mathrm{km}$, taxi drivers often chose the shortest distance to arrive destination, and drivers will almost not try to search customers on the way.

Traditionally, empty taxi drivers often search customers by setting original route and if no customer will be found in this route, the drivers will re-set the searching route until find new customers. Owing to the long-term market regulation, the empty taxi drivers often driver to areas like downtown and train station with multiple customers. But taxi apps change route choices for empty taxi hugely, offering equal information to customers and drivers both. Taxi drivers will be able to receive targeting orders benefit themselves and lower the cost for empty driving.

\section{Subsidy influences to taxi order receiving}

If the customer amount is without limits in taxi market, the starting price for taxi will influence the enthusiasm, which also means order receiving amount for drivers. The taxi price is composed of 
two parts, starting price and mileage price calculating via distance. Generally, the upsurge of starting price will increase the unit price for taxi order and add income for drivers.

(1) Subside by order numbers

Introduce the operation subsidy for drivers $b_{1}$, suppose to subside by $b_{1} \mathrm{RMB} /$ order, and the income $I_{0}^{\prime}(x)$ for per mile/order

(2) Subside by mileage

Introduce the operation subsidy for drivers $b_{1}{ }^{\prime}$, suppose to subside by $b_{1}{ }^{\prime} \mathrm{RMB} / \mathrm{km}$, and the income $I_{0}^{\prime \prime}(x)$ for per mile/order

\section{Subsidy Influences to Supply and Demand Relationship}

\section{Total revenue for taxi based on time pricing}

In the actual situation,taxi supply is related to actual price of taxi service at that time and the distribution of demand for taxi in previous moment. Taxi price will not change in a long term, the actual payment from customer only relates to the subsidy amount at that time, demand of taxi service shall not influenced by that of previous moment, but affected by that of this time.

Suppose the taxi speed v constant, time interval of each order is a constant $t^{\prime}$, the profit of per taxi order without considering of subsidy will be $F_{t}=f_{d} \cdot d=f \cdot v \cdot t=(f \cdot v) \cdot t, \mathrm{~d}$ is regarded as the driving distance of any taxi; $f_{d}$ expresses the average price of unit distance; expresses the price of unit time under the condition of $f_{t}=f \cdot v$, that is pricing by time, this expression can be transferred to the relationship between profit of any taxi order

(1) Subside by fix amount for per order

The total order for taxi in a time range express as $\theta$ and total revenue express as $Z$, that is $Z=\sum_{n=1}^{\theta}\left[F_{\theta}(t)+b_{1}\right]$

Introduce $F_{\theta}(t)=f_{t} \cdot t(\theta)$, to get the result of $Z=\sum_{n=1}^{\theta}\left(f_{t} \cdot t(\theta)+b_{1}\right)=\left(b_{1}-f_{t} \cdot t^{\prime}\right) \cdot \theta+f_{t} \cdot T$

If $b_{1}-f_{t} \cdot t^{\prime}>0$, that is $b_{1}>f_{t} \cdot t^{\prime}, f_{t} \cdot t^{\prime}$ express as the lose amount in empty driving intervals $t^{\prime}, b_{1}$ express as subsidy amount to drivers for per order. The income of drivers is $Z$, which will increase with order numbers $\theta$. Thus drivers would be pron to receive short term order not the long term one and use $b_{1}$, subsidy amount to drivers for per order to cover the interval lose of empty driving. The pressure of taxi-call difficulties will be remission with the balance of supply and demand for short term orders.

(2) Subside by mileage pricing

$Z=\sum f_{t} \cdot t(\theta)+t(\theta) \cdot v \cdot b_{1}=\left(f_{t}+v \cdot b_{1}\right) \cdot\left(T-t^{\prime} \cdot \theta\right)$

The total service time for taxi must be longer than that of empty driving, that is $T-t^{\prime} \cdot \theta>0$, if $t^{\prime}$ is constant, $\left(T-t^{\prime} \cdot \theta\right)$ would decrease with $\theta$ increases, thus the total income $Z$ for drivers would decrease, too.

So drivers would be prone to receiving long term order not short term one and use $b_{1}$, subsidy amount to drivers for per order to cover the interval lose of empty driving. This will make the supply and demand of short term orders more unbalance and shall not benefit taxi-call problem solving.

\section{Evolutionary game theory model}

To apply the principle of evolutionary game theory, the formation process of subsidy and bi-lateral behaviors between drivers and customers is the constant adaptation process between the two sides, and the apps only adopt the subsidy accepted by one side will come to dissapper. In the description of classic game theory, Nash equilibrium suppose the two side in game are both entirely 
rational, but that is not impossible in actual condition. We can suppose the taxi drivers and customers are both bounded rational, they need to keep adapting each other and study to improve the strategy to reach balance and make the final choice for new scheme.

Suppose D as the set of taxi drivers, $\mathrm{P}$ as the set of customers.

Stipulate the strategy sets of taxi drivers as $\left\{S_{m}=\right.$ only receive short term $\operatorname{orders}\left(S_{m 1}\right)$, only receive long term orders $\left.\left(S_{m 2}\right)\right\}$, and suppose the $S_{m 1}$ ratio in $S_{m}$ as $x$, so $S_{m 2}$ ratio in $S_{m}$ is $(1-x)$;

Stipulate the strategy sets of customers as $S_{n}=\left\{\right.$ only choose other transportation $\left(S_{n 1}\right) \quad$, only choose taxi for short term trips $\left(S_{n 2}\right)$, only choose taxi for long term trips $\left.\left(S_{n 3}\right)\right\}$, Suppose $S_{n 1}, S_{n 2}, S_{n 3}$ ratio in $S_{n}$ is $\left(1-Y_{1}-Y_{2}\right), Y_{1}, Y_{2}$. Suppose the utility of one trip for customers as: $V_{i}=\alpha_{i}+\beta_{i} \cdot F_{i}+\gamma_{i} \cdot T_{i}$

The taxi driver has a $\pi$ profit per kilometer,

$\pi=$ Expenses for passengers $F\left(F_{i}, i=1,2\right)$ - $\operatorname{costs} q\left(q_{i}>0, i=0,1,2\right)$

When the passengers go by other means of transport, taxi in no-load consumption cost, the profit can be expressed as $\pi_{0}=-q_{0}$; if every single fixed amount of subsidies, taxi drivers take profits $\pi_{1}=F_{1}-q_{1}+b_{1}$ for short distance passenger taxi driver; adhere to long-distance passenger profits $\pi_{2}=F_{2}-q_{2}+b_{2}$; if the mileage subsidies, taxi drivers to take short trips profits $\pi_{1}=F_{1}-q_{1}+b_{1} x$ for the long-distance passenger taxi driver; adhere to the profits $\pi_{2}=F_{2}-q_{2}+b_{2} x, \quad x$ which indicates an operating mileage.

The payment matrix of the taxi group and the passenger group is as follows:

Table 1

\begin{tabular}{ccc}
\hline \multirow{2}{*}{ Passenger } & \multicolumn{2}{c}{ Taxi } \\
\cline { 2 - 3 } & $S_{m 1}$ & $S_{m 2}$ \\
\hline$S_{n 1}$ & $V_{0}, \pi_{0}$ & $V_{0}, \pi_{0}$ \\
$S_{n 2}$ & $V_{1}, \pi_{1}$ & $V_{0}, \pi_{2}$ \\
$S_{n 3}$ & $V_{0}, \pi_{1}$ & $V_{2}, \pi_{2}$ \\
\hline
\end{tabular}

According to the above conclusion, the dynamic equation of the taxi drivers' population is derived:

$$
X=\frac{d X}{d t}=X\left[U_{m 1}-R_{m}\right]=X\left[\left(Y_{1}+Y_{2}\right)\left(\pi_{1}-\pi_{2}\right)(1-X)-X\right]
$$

Average revenue per passenger travel

$$
R_{n}=V_{0}-\left(Y_{1}+Y_{2}\right) \cdot V_{0}+\left(Y_{1}+Y_{2}\right) \cdot\left(V_{1}+V_{0}\right) \cdot X+(1-X) \cdot\left(Y_{1}+Y_{2}\right) \cdot\left(V_{0}+V_{2}\right)
$$

According to the conclusion of the above conclusion, the dynamic equation of the travel passenger population is deduced.

$$
Y_{1}+Y_{2}=\frac{d\left(Y_{1}+Y_{2}\right)}{d t}==\left(Y_{1}+Y_{2}\right)^{2}\left[V_{0}-\left(V_{1}+V_{0}\right) \cdot X-(1-X)\left(V_{0}+V_{2}\right)\right]
$$

Analysis of the dynamic equation of the travel passenger population, $Y_{1}+Y_{2}=\frac{d\left(Y_{1}+Y_{2}\right)}{d t}=0$. When $X>\frac{V_{2}}{V_{2}-V_{1}}, X^{*}=0$ and $X^{*}=1$ are the two stable states, in which $X^{*}=0$ is the evolutionary stability strategy; when $X<\frac{V_{2}}{V_{2}-V_{1}}, X^{*}=1$ and $X^{*}=0$ are the two stable states, $X^{*}=1$ which is the evolution of the stability strategy. 


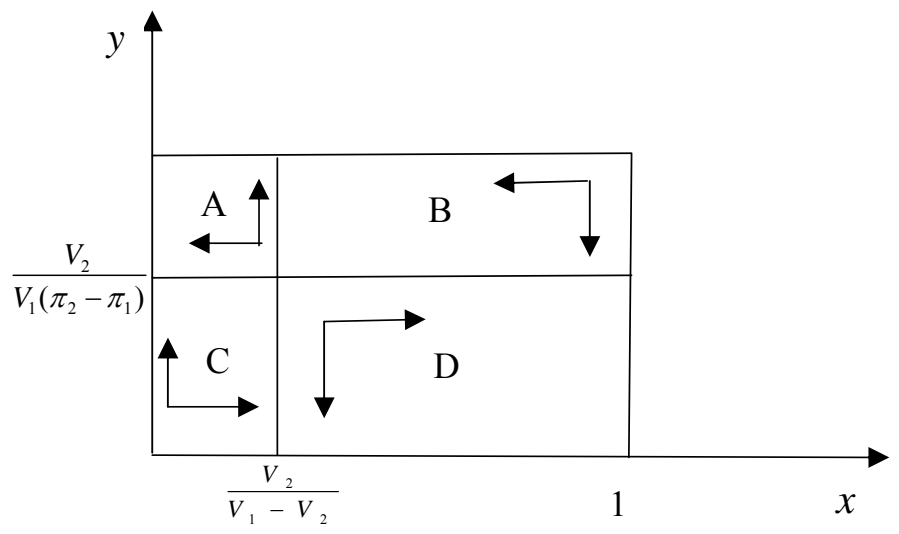

Figure 1

If you choose a subsidy for each knowable, $\pi_{2}-\pi_{1}<0, V_{2}>V_{1}>0$ choose a taxi number will rise, because $V_{1}$ the bus utility is constant, when $V_{2}$ the rise is short, $\frac{V_{2}}{V_{1}}$ utility increases, increase drivers tend to pick a single short.

If you choose to set per kilometer, $\pi_{2}-\pi_{1}>0, V_{1}>V_{2}>0$ choose a taxi subsidies, the number will rise at the same time, because $V_{1}$ the bus utility is constant, when the $V_{1}$ drop is long utility increases, $\frac{V_{2}}{V_{1}}$ increase drivers tend to be more changduantu single.

\section{Practical situation analysis}

(1) Data selection

Select a city one day taxi distribution as the specific case analysis and distribution as shown below:

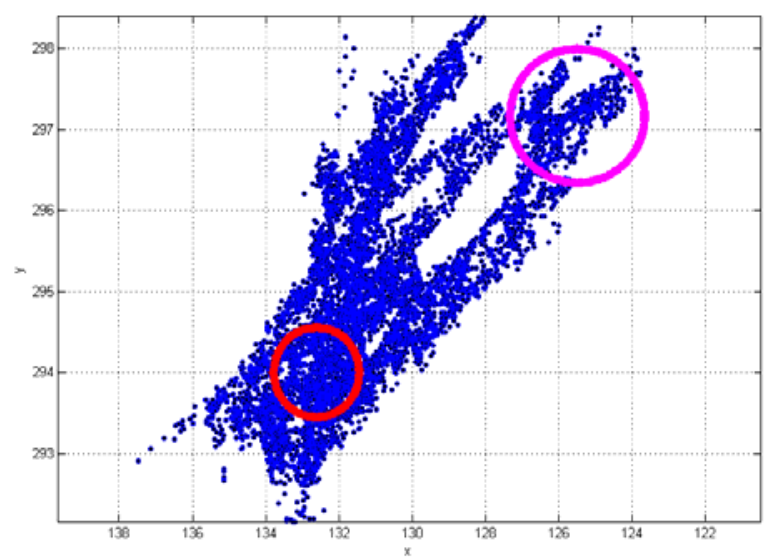

Figure 2

Select two representative areas of suburban area (pink) and central area (red).

Select the most low and late afternoon peak period of representative time: 4:00 - 6:00 (valley), 9:00 - 11:00 (peak) and 16:00 - 18:00 - (sub peak) of these three time periods: 


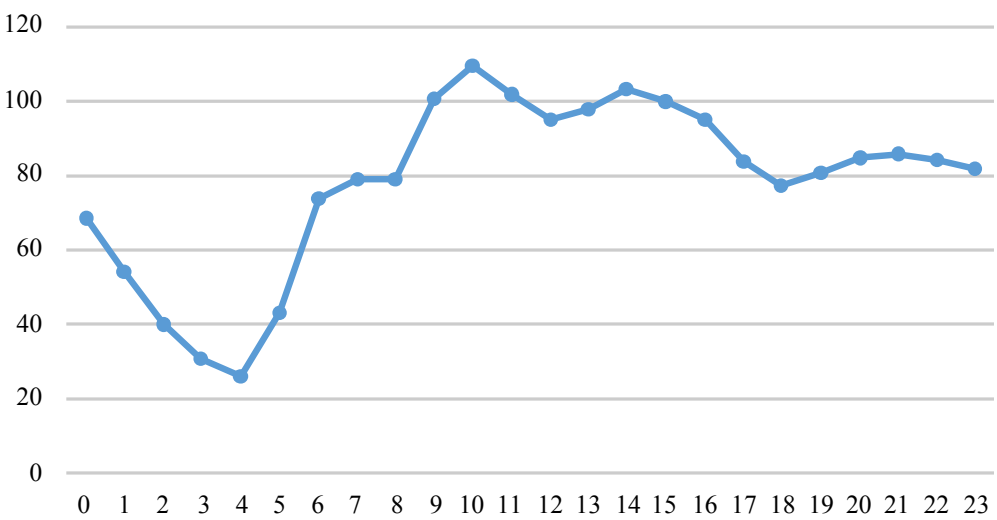

Figure 3

Select representative time

The basic relationship between supply and demand model by passenger taxi, calculation of Xinjiekou area and Xianlin University City area density, taxi taxi Kongshi rate and the average waiting time of the corresponding value respectively, as shown in the following table:

Table 2 Evaluation index data in the central area

\begin{tabular}{cccc}
\hline \multirow{2}{*}{ Indictor } & \multicolumn{3}{c}{ Time } \\
\cline { 2 - 4 } & $4: 00-6: 00$ & $9: 00-11: 00$ & $16: 00-18: 00$ \\
\hline Taxi density & 0.84 & 0.91 & 0.89 \\
The taxi empty rate & 0.025 & 0.96 & 0.96 \\
waiting time & 0.95 & 0.75 & 0.66 \\
\hline \multicolumn{3}{c}{ Table 3 Evaluation index data in suburban areas } \\
\hline \multicolumn{3}{c}{ Time } \\
\cline { 2 - 4 } Indictor & $4: 00-6: 00$ & $9: 00-11: 00$ & 0.79 \\
Taxi density & 0.77 & 0.93 & 0.79 \\
The taxi empty rate & 0.025 & 0.98 & 0.66 \\
waiting time & 0.97 & 0.75 &
\end{tabular}

\section{Amendments to the Subsidy Scheme}

In order to use a taxi software to make social traffic resources more reasonable and more reasonable, for the coordination of the relationship between people and cars, the total amount of subsidies to the total amount of subsidies allocated. Taking into account the driver subsidies are conducive to improving the initiative to reduce the driver no load time, reduce the waiting time for passengers, the passenger subsidies are conducive to new customers to join, so as to facilitate the public to travel while increasing the driver's income, so reasonable subsidy allocation is particularly important. Therefore, using the relationship between subsidies and waiting time, combined with the theory of welfare society in economics, quantitative discussion is of the distribution of subsidies so that the driver's income increased, making customers enjoy preferential and waiting for the time to be able to accept the range.

Taxi drivers subsidies, drivers operating costs down, the driver will tend to pick more single, so to meet the needs of passengers, passengers can wait in the ideal car time to wait for the passengers which will save time costs, ease the taxi difficult problem, to a certain extent, improving customer demand. Market demand is still waiting for the time to change, at this time to establish a taxi service supply and demand balance, considering the elasticity of the market demand for taxi operators and taxi in the equilibrium conditions. it found that the price of subsidies and the same to change and that the price of subsidies and reverse may change. The driver of the car subsidy means to improve the efficiency of the driver, so that passengers can spend less time on the cost, indirectly reducing 
the taxi rate and increase the taxi driver's income, which can form a virtuous circle.

If a taxi platform at the same time for both drivers and passengers to carry out subsidies, the impact of the economy in the demand curve $D$ and supply curve $S$ at the same time to move to a straight line $D^{\prime}$ and $S^{\prime}$.

The intersection $P$ of each line and the axis is from top to bottom $b_{a}, b_{b}, b_{c}, b_{d}, b_{e}, b_{f}$. The slope $k_{S}$ of the line $S, S^{\prime}$ is linear $D, D^{\prime}, k_{D}$ since

Social welfare change after subsidy= consumer surplus increase-total amount of the platform subsidy $=-\triangle$ KEL square $=-0.5\left(\mathrm{t}+\mathrm{t}^{\prime}\right) *$ increased demand

Therefore, $\frac{\left(b_{a}+b_{c}\right)-\left(b_{d}+b_{f}\right)}{k_{s}-k_{d}}=1$ is the optimal distribution formula.

\section{Conclusion}

The reason why there are difficult taxi-hailing problem in City is mainly that the taxi driver is more inclined to receive a higher return than a long single and not willing to receive a small income, but passengers travel mainly short single. Each taxi software platform launched a different taxi subsidy program to provide a variety of ways to adjust the level of taxi supply and demand. But the subsidies in the market of several common analysis shows that whether the subsidy per unit or the number of kilometers of subsidies, which can not fundamentally solve the problem of difficult to take a taxi, taxi software companies should be based on big data since the operation to reconsider the subsidy plan scientifically so as to serve the public.

\section{Reference}

[1] Song Haiqing, new energy vehicle subsidy policy research

[2] Chen Feng, a reasonable scale of urban taxi based on supply and demand balance,

[3] Deng Yuanhui Ou Guoli, Beijing city taxi use evaluation and analysis, comprehensive transportation efficiency, volume number: 2009 (06), 54-592009

[4] Urban Transport of China, Deng Zhongwei, Ji Minhe. Shanghai city taxi distribution of [1672-5328 (2012) 01-0068-07]. Urban Transport of China, 2012, Vol.10, No.1:69-70. Journal of Traffic and Transportation Engineering.

[5] Wang Wei, Lu Jian. [1671-1637 01-0093-06]. of (2007) Journal Traffic Engineering Transportation and, 2007, Vol.7:94-96.

[6] master's degree thesis of Beijing Jiaotong University - the dragon. Influence of taxi price adjustment on the morning Kotewall residents travel sensitivity of [1004]. master's degree paper of Beijing Jiaotong University, 2015, Vol.1:19-25. Journal of Anqing Teachers College. 